\title{
Zwischen Naturerklärung und Naturferne - Interpretationen und Praxis der Synthetischen Biologie $^{\star}$
}

Elisabeth Loos

Der Begriff der Natur ist das leitende Thema im vorliegenden Band, der damit in ein vielschichtiges Diskussionsfeld der Geistes- und Naturwissenschaften vorstösst. Die ohnehin komplexe Begriffsgeschichte, die zu grossen Teilen von der Philosophie und Theologie bestimmt wurde, wird in jüngerer Zeit um fast schon handwerklich anmutende Fragen ergänzt, die sich aus den Forschungen der angewandten Naturwissenschaften ergeben: Gelingt es dem Menschen tatsächlich, zum Baumeister der Natur zu werden, indem er die Naturprozesse nicht nur zu verstehen sucht, sondern sie in der Synthese zur Anwendung bringt? Angesprochen ist damit die Natur im Sinne des naturwissenschaftlichen Forschungsobjekts, das weniger durch explizite Definitionen etwa von den geisteswissenschaftlichen Forschungsobjekten abgegrenzt wird, als vielmehr durch die faktisch bestehenden Disziplinen (Physik, Chemie, Biologie, Astronomie, Geologie etc.) und ihre methodischen Ausrichtungen umrissen werden kann. Als ein Bereich darin kann die belebte Natur bestimmt werden, deren Erforschung seit gut zweihundert Jahren in der Biologie angesiedelt wird. Um die Bemühungen, die belebte Natur oder zumindest Teile daraus im Labor zu synthetisieren, soll es im Folgenden gehen, genauer gesagt um die sogenannte Synthetische Biologie. Nach einer kurzen Vorstellung dieser Forschungsrichtung soll der Blick auf verschiedene Interpretationen des Verhältnisses der Synthetischen Biologie zur (belebten) Natur gerichtet werden. Das Anliegen des Aufsatzes ist es, die Verortung der Synthetischen Biologie zwischen "Naturferne« und "Naturerklärung" aufzugreifen, um zunächst einmal auf die unterschiedlichen Interpretationsmöglichkeiten von "Natur" im Kontext der synthetisch-biologischen Forschungen hinzuweisen. Eine daraus ableitbare, provokante Lesart

\footnotetext{
* Dies ist die leicht veränderte Version eines Vortrages, der am 28.9.2018 im Rahmen der Tagung "Religion and Science - Friend or Foe or Unrelated? « am Institut für Hermeneutik und Religionsphilosophie der Universität Zürich gehalten wurde. Ich danke den Diskussionsteilnehmerinnen und -teilnehmern für ihre Rückfragen und Anmerkungen zum Thema.
} 
der Synthetischen Biologie ist, in ihren Fortschritten die Einlösung des reduktionistischen Versprechens der naturwissenschaftlichen Letzterklärung der belebten Natur zu sehen. Zu Recht werden solche reduktionistischen Deutungen, die ja nicht nur im Kontext der Synthetischen Biologie auftauchen, als metaphysische und damit den Rahmen der naturwissenschaftlichen Methode sprengende Aussagen entlarvt. Die These, die ich in diesem Aufsatz vertrete, ist jedoch, dass der (oftmals geisteswissenschaftliche) Reflex, in die Diskussion um die neueren Entwicklungen etwa der Biotechnologie mit einer grundlegenden Reduktionismuskritik einzusteigen, dem pragmatischen Charakter der naturwissenschaftlichen Forschungen zu wenig Beachtung schenkt.

\section{Die Synthetische Biologie}

Was ist Synthetische Biologie? Als erste Annäherung an dieses vergleichsweise junge Forschungsfeld soll hier die Beschreibung dienen, dass die Synthetische Biologie das Projekt "der gezielten Konstruktion von lebenden Biomaschinen aus standardisierten Komponenten " ${ }^{1}$ umfasst. Bekannt aus den Medien sind vor allem die Forschungen des "John Craig Venter Institute" (JCVI), die ich zur Illustration des Forschungsfeldes heranziehen möchte: 2010 wurde die erste sogenannte "synthetische Zelle» vorgestellt, ${ }^{2}$ deren komplettes Genom in silico designt und in vitro synthetisiert wurde, bevor sie in eine entkernte Spenderzelle eingebracht wurde: "The first self-replicating species we've had on the planet whose parent is a computer.", wird Craig Venter dazu zitiert. ${ }^{3} 2016$ stellte das JCVI dann eine weitere synthetische Zelle vor, die die bisher kleinstmögliche Zahl an Genen enthält und als "working approximation" an

\footnotetext{
1 Simon Ausländer/David Ausländer/Martin Fussenegger, Synthetische Biologie die Synthese der Biologie, Angewandte Chemie 129 (2017), 6494-6519, hier 6495.

2 In der Publikation des Fachartikels wird die umstrittene Bezeichnung "synthetic cell« damit gerechtfertigt, dass die phänotypischen Effekte der Zelle massgeblich von dem chemisch synthetisierten Genom bestimmt würden und nicht vom Spendercytoplasma, siehe Daniel G. Gibson et al., Creation of a Bacterial Cell Controlled by a Chemically Synthesized Genome, Science 329 (2010), 52-56, hier 56. Kritisiert wird die Bezeichnung "synthetic cell « unter anderem von Steen Rasmussen in einer Kurzstellungnahme, Life after the synthetic cell, Nature Opinions 465 (2010), 422-424, hier $422 f$.

3 https://www.nytimes.com/2010/05/21/science/21cell.html (6.2.2019).
} 
eine Minimalzelle bezeichnet wurde. ${ }^{4}$ Dass es nur eine "working approximation" ist, liegt vor allem daran, dass sich 150 Gene mit unbekannter Funktion als unverzichtbar für die Fast-Minimalzelle erwiesen haben und daher nicht "wegminimalisieren « liessen. Solche Minimalzellen sind für die Synthetische Biologie von Bedeutung, weil die Reduzierung der genetischen Komplexität mit einer verbesserten Kontrolle der Zellreaktionen einhergeht und somit die Vision einer "lebenden Biomaschine" mit kontrollierbaren, "vordefinierten Aufgaben « ${ }^{5}$ ein Stück weit näher rückt. ${ }^{6}$

Es gibt jedoch - wie so oft bei Überbegriffen dieser Art - keine einheitliche Definition der Synthetischen Biologie. Das hängt unter anderem damit zusammen, dass unter dem Label "Synthetische Biologie« sehr verschiedene Ansätze gesammelt werden, die von den erwähnten Projekten der Genomsynthese und der Minimalisierung des Genoms über das Design und die Synthese von Stoffwechselkreisläufen bis hin zu xenobiologischen Projekten wie der Implementierung künstlicher Nukleinsäuren (XNA) ins Genom reichen.

\section{Die Synthetische Biologie und ihre Bezugnah- me auf »die Natur « und »das Natürliche»}

Darüber hinaus ist bereits umstritten, ob die Synthetische Biologie tatsächlich von der klassischen Gentechnologie abzugrenzen und als neuartige Forschungskategorie zu definieren ist, d.h. ob man die Synthetische Biologie als Unterart der Gentechnologie, als ihre Weiterentwicklung oder als eigenständige Denk- und Forschungslinie begreifen soll. ${ }^{7}$ Davon wird auch die Verhältnisbestimmung der Synthetischen Biologie zur Biologie und deren Forschungsgegenstand beeinflusst - womit die Frage nach dem Verhältnis zur (belebten) "Natur« und »dem Natürlichen« angesprochen ist, die im Folgenden aufgegriffen wird.

4 Clyde A. Hutchison III et al., Design and synthesis of a minimal bacterial genome, Science 351 (2016), aad 6253, hier aad 6253-1.

5 Ausländer et al., Synthetische Biologie, 6495.

6 Genauer gesagt sollen die Minimalzellen die Funktion eines "chassis", d.h. eines Zellgerüsts, erfüllen, das mit verschiedenen modularisierten Merkmalbausteinen zur Wunschzelle ausgebaut werden kann - dies ist zumindest die Vision.

7 Dieses Thema nimmt zum Beispiel ein Themenband der Berlin-Brandenburgischen Akademie der Wissenschaften (BBAW) aus dem Jahr 2012 als Leitfrage auf, s. Kristian Köchy/Anja Hümpel (Hg.), Synthetische Biologie. Entwicklung einer neuen Ingenieurbiologie?, Themenband der interdisziplinären Arbeitsgruppe Gentechnologiebericht, Dornburg 2012. 


\subsection{Naturferne}

Die »klassische« Gentechnologie wird etwa vom J. Craig Venter Institute in einem scharfen Kontrast zu den eigenen Ansätzen gesehen, da jene nur modifizierend in natürliche Genome eingreife, man selbst aber vollständig synthetisierend vorgehe. ${ }^{8}$ Die Argumentation zielt darauf $\mathrm{ab}$, dass es einen prinzipiellen Bruch zwischen natürlichen, wenn auch modifizierten Genomen und komplett im Labor synthetisierten, d.h. unnatürlichen Genomen gebe; um die eigene Leistung hervorzuheben, werden die synthetisierten Genome in einen scharfen Gegensatz zu den natürlich entstandenen - im Sinne des "omne vivum ex ovo « 9 - gesetzt und gerade die Naturferne der Synthetischen Biologie hervorgehoben. Es mutet allerdings willkürlich an, dass allein die Art der materiellen Entstehung des Genoms als Kennzeichen für die Natürlichkeit bzw. Künstlichkeit/ Neuartigkeit einer Zelle dienen soll. Ausserdem ist festzuhalten, dass die Eingriffstiefe ins Genom kontinuierlich gewachsen ist und somit kein prinzipieller Bruch zwischen der Synthetischen Biologie und der übrigen, die natürlichen Zellen "nur« modifizierenden Biotechnologie behauptet werden kann. ${ }^{10}$

Neben der Tiefe des Eingriffs in das Genom wird noch ein weiteres Charakteristikum der Synthetischen Biologie häufig genannt, im Bemühen, das Spezielle ihrer Arbeitsweise zu erfassen: das "engineering ideal«, d.h. der starke Bezug der Synthetischen Biologie auf die Ingenieurwissenschaften. Diesen Punkt möchte ich hier aufgreifen, da er in der synthetisch-biologischen Fachliteratur und in der Sekundärliteratur immer wieder betont wird; wenn man sich beim Versuch, die Synthetische Biologie zu definieren, auf etwas einigen kann, dann gehört die Orientierung an den Paradigmen der Ingenieurwissenschaften auf jeden Fall dazu. ${ }^{11}$ Auch hier wird die "Natur" als etwas konstruiert, von dem man sich wegbewegt, indem die Unkontrollierbarkeit der natürlichen Organismen in die Kon-

\footnotetext{
8 Gibson et al., Creation of a Bacterial Cell, 55.

9 Dass dieses Prinzip im Zeitalter der Synthetischen Biologie nicht länger gelte, ist zum Beispiel die Meinung des Molekularbiologen David Deamer, der 2010 im Magazin Nature um eine Kurzeinschätzung der »synthetischen Zelle« Craig Venters gebeten wurde, siehe Life after the synthetic cell, 424 .

${ }^{10}$ Dies unterstreichen auch mehrere Forscher, die ebenfalls vom Magazin Nature nach ihrer Einschätzung der "synthetischen Zelle» gefragt wurden: So bewertet der Genetiker George Church die entstandene Zelle eindeutig nicht als "new life» und Martin Fussenegger betont, dass es sich um "a technical advance, not a conceptual one» handle. Siehe Life after the synthetic cell, 422-424.

11 Ausländer et al., Synthetische Biologie, 6495.
} 
trollierbarkeit von Biomaschinen überführt werden soll. Schon die genannte Beschreibung der Synthetischen Biologie als Vorhaben der "gezielten Konstruktion" von Maschinen - Biomaschinen zwar, aber Maschinen - aus "standardisierten Komponenten" führt gedanklich eher in die Werkstatt als in die Natur. Standardisierung, Modularität und Vereinfachung der Komponenten sind die angestrebten Kennzeichen der synthetischen Zelle, und der methodische Königsweg dahin ist das "rational design". Die Steuerung von synthetischen Stoffwechselwegen über logische Operatoren und das gezielte Anund Ausschalten von Genexpression über synthetische Genschalter ("toggle switch«) ermöglichen die Kontrolle immerhin einzelner Zellkomponenten, wenn auch noch nicht der Gesamtheit der Zellreaktionen. Die Minimalzellforscherin Petra Schwille vergleicht die natürlichen Organismen mit "Rube-Goldberg-Maschinen", die ihre Aufgaben zwar verlässlich, aber in unnötig komplizierten und zum Teil redundanten Schritten ausführen; die natürliche Evolution habe durch planloses Rumbasteln, »tinkering«, diese Wege hervorgebracht, deren Ziel man aus Sicht der Synthetischen Biologie auch einfacher erreichen können müsse - das »tinkering « der natürlichen Evolution, das Schwille mit Bezug auf François Jacob ${ }^{12}$ aufgreift, soll in der Minimalzelle durch "engineering " ersetzt werden. ${ }^{13}$ Die dahinterstehende ingenieurwissenschaftliche Denkweise kann mit einem in der Forschungsszene bekannten Witz verdeutlicht werden: »The biologist goes into the laboratory in the morning and she discovers that the system she's looking at is two times as complicated as she thought it was. > Great! $<$ she says, $>$ I get to write a paper. $<$ The engineer goes into the lab, gets the same result and says, >Damn. How do I get rid of that? « ${ }^{14}$ Die Erforschung des Bestehenden, der Natur mit all ihren ineffizienten und komplizierten Prozessen, wird der Biologie zugeschrieben, während der ingenieurwissenschaftliche Blick auf die Vereinfachung der Prozesse gerichtet ist - ohne dass damit zwangsläufig das Ergebnis der Prozesse anders ausfallen muss. ${ }^{15}$ In diesem Kontext gilt als Kennzeichen der "natürlichen" belebten $\mathrm{Na}-$ tur die ungerichtete Entstehungsgeschichte durch das evolutionäre "tinkering", während die Naturferne der synthetisch-biologischen

\footnotetext{
12 François Jacob, Evolution and Tinkering, Science 196 (1977), 1161-1166.

${ }^{13}$ Petra Schwille, Bottom-up Synthetic Biology: Engineering in a Tinkerer's World, Science 333 (2011), 1252-1254, hier 1253.

${ }^{14}$ S. https://www.wired.com/2008/01/biology-moving/ (6.2.2019).

15 "From such a perspective, the underlying question of synthetic biology would not be how a biological system actually functions, but rather, how it could in principle function with a minimal set of elements." Schwille, Bottom-up Synthetic Biology, 1253.
} 
Systeme durch ein Zusammenstreichen der als ineffizient betrachteten Prozesselemente definiert wird, das in einer verbesserten Kontrolle mündet.

\subsection{Naturerklärung durch Natursynthese}

Zugleich zeigt sich bei aller angestrebten und demonstrierten Emanzipation von der Natur, dass die Bezugnahme auf das Natürliche nicht nur mit dem Ziel der Abgrenzung stattfindet, sondern auch, um die Beherrschbarkeit des Natürlichen zu demonstrieren. Auch dieser Aspekt steht im Zusammenhang mit einer technischingenieurwissenschaftlichen Perspektive auf das Lebendige. Diese Perspektive ist freilich keine Erfindung des 21. Jahrhunderts und selbst die konkreten Grundgedanken der Synthetischen Biologie können bis ins 19. Jahrhundert zurückverfolgt werden. ${ }^{16}$ Dort findet sich beim Biologen Jacques Loeb auch der Gedanke, dass die Biologie ihren Gegenstand erst wirklich erfassen könne, wenn sie in der Lage sei, ihn zu synthetisieren; erst mit dem Eintritt der Biologie in ihre "synthetische Phase" sei demnach ein wirklich wissenschaftlicher Status erreicht. Idealisiertes Vorbild ist dabei die Chemie, deren historische Entwicklung in drei Phasen beschrieben wurde: beschreibend, analytisch und synthetisch, wobei die letztgenannte Phase oftmals festgemacht wird an der gelungenen Synthese eines organischen Stoffs aus anorganischen Ausgangsstoffen durch Friedrich Wöhler 1828, nämlich Harnstoff, den man zuvor nur aus Urin gewinnen konnte. "What I cannot build, I cannot understand", so der passende Sinnspruch, den im Jahr 2010 die Wissenschaftlerinnen und Wissenschaftler des J. Craig Venter Institute in die Basenabfolge der bereits erwähnten »synthetischen Zelle« encodiert haben. ${ }^{17}$ Mit der gelungenen Konstruktion eines Lebewesens im Labor lasse die Biologie die reine Beschreibung und Analyse hinter sich und trete ein in ihre synthetische Phase, die als einzige ein wahres Verständnis der Grundlagen der Biologie - und damit der belebten Natur - ermögliche, so die dahinterstehende Logik.

Es werden also zwei Aspekte angesprochen, wenn es um das Verhältnis der Synthetischen Biologie zum "Natürlichen« geht. Zum

\footnotetext{
${ }^{16}$ Eine Übersicht zur Geschichte der Synthetischen Biologie gibt Heiner Fangerau, Zur Geschichte der Synthetischen Biologie, in: Synthetische Biologie, hg. v. Kristian Köchy et al., 61-84.

${ }^{17}$ Der Ausspruch wird meist auf Richard Feynman zurückgeführt und wurde im synthetischen Genom verschlüsselt, s. https://singularityhub.com/2010/05/24/venters-newest-synthetic-bacteria-has-secret-messages-coded-in-its-dna/ (6.2.2019).
} 
einen steht die Distanzierung von der Natur, sogar ihre Überbietung im Vordergrund, indem eine technisch beherrschbare Version der Lebewesen angestrebt wird, die von natürlichen Organismen inspiriert ist, aber gerade in ihrer Naturferne das synthetisch-biologische Profil bestimmt. In diesem Fall ist es gerade die »Künstlichkeit» der synthetisch-biologischen Systeme, die als angestrebtes Ziel formuliert wird und so die Konstruktion eines ursprünglichen, unberührten und unkontrollierbaren "Natürlichen" als Gegenpol erlaubt. Diese Emanzipation von der Natur findet Ausdruck in einem berühmt gewordenen Ausspruch: »The genetic code is 3.6 billion years old. It's time for a rewrite. ${ }^{18}$ Mit Blick auf die Einordnung der Synthetischen Biologie in den Fächerkanon derWissenschaften liegt es hier nahe, sie eher im Umfeld der Ingenieurwissenschaften zu verorten und sie als Abkopplung der Biologie zu betrachten.

Zum anderen können die Forschungen der Synthetischen Biologie für eine weitere, auf den ersten Blick gegensätzliche Interpretation herangezogen werden, nach der die Synthetische Biologie mittels ihrer Synthesekompetenz zu einer Letzterklärung der belebten Natur führen wird. Findet die Naturwissenschaft, vor allem die Biologie, endlich in ihrer "synthetischen Phase $z u$ einem wahrhaft wissenschaftlichen Verständnis ihres Gegenstandes? Dies käme einer Annäherung der Wissenschaft an ihren Gegenstand gleich. Diese Sichtweise wird vertreten, wenn die Entwicklung der Synthetischen Biologie als Weiterentwicklung der Biologie interpretiert wird, parallel zur historischen Entwicklung der Chemie, wie oben erläutert wurde. Das Wissen zur Synthese wird dann gleichgesetzt mit dem Wissen um die Beschaffenheit und Funktionsweise der belebten Natur. Eine Auswirkung dieser Sichtweise wäre, die Synthetische Biologie als eine besonders fortschrittliche Unterdisziplin innerhalb der Biologie anzusehen, quasi als die Vollendung des biologischen Wissens.

Je nach Narrativ kann eine der beiden Interpretationen in den Vordergrund rücken und beide provozieren auch kritische Rückfragen. Zum einen muss festgehalten werden, dass die Synthetische Biologie von einem vollständigen Verständnis der belebten Natur sehr weit entfernt ist - man muss nicht einmal auf die Komplexität des menschlichen Gehirns verweisen, um diesen Punkt zu verdeutlichen, sondern kann es bei einer einfachen Zelle belassen.

Andererseits muss auch die Hervorhebung der Emanzipation von der Natur durch die Synthetische Biologie eingeschränkt werden,

${ }^{18}$ Der Ausspruch wird auf Tom Knight vom Massachusetts Institute of Technology (MIT) zurückgeführt. 
denn bei allen Versuchen, neuartige und historisch gesehen noch nie dagewesene und deshalb als künstlich und fremd bezeichnete Komponenten $\mathrm{zu}$ entwerfen (XNA etc.), bleibt das konzeptuelle Vorbild des historisch gewachsenen irdischen Lebens klar bestehen. Die synthetischen Varianten der natürlichen Vorbilder können somit eher als Imitationen oder Reduktionen denn als wirkliche Neuerfindungen angesehen werden.

Es ist erst die Zusammenschau der beiden Aspekte, die zu einem reduktionistischen Verständnis der belebten Natur führt, das als metaphysische Übersteigerung der naturwissenschaftlichen Forschung angesehen werden muss.

Denn tatsächlich sind die beiden Deutungen nur oberflächlich betrachtet widersprüchlich und können leicht kombiniert werden. Dann liegt das Bild der Lebewesen zugrunde, dass die Reduktion auf das Kontrollierbare dem eigentlichen - natürlichen - Kern der natürlichen Lebewesen entspricht. Die bessere Erforschung der Natur soll auf der Grundlage einer Emanzipation von den natürlichen Vorbildern stattfinden. Der »tinkering«-Anteil wird zum Ballast, der nicht nur in der technischen Imitation der Lebewesen aussortiert wird, sondern auch als verzichtbar für Lebewesen schlechthin angesehen wird. Das Kontingente, Ineffiziente, durch Basteln Hervorgebrachte, die Redundanz der Reaktionswege ist evolutionär nachvollziehbar, aber funktional unnötig.

Die genannte Minimalisierung der Zelle spielt eine wichtige Rolle in dieserVision der Synthetischen Biologie, denn mit ihr soll realisiert werden, was bisher nicht möglich ist, nämlich die Funktion jedes einzelnen Gens zu bestimmen, das notwendig für "Leben" sei: "This facilitates the goal to achieve an understanding of the molecular and biological function of every gene that is essential for life. ${ }^{19}$ Bisweilen wird mit den Forschungen der Synthetischen Biologie die explizite Hoffnung verbunden, dass gerade diese Vereinfachung und Rationalisierung der Organismen "a new understanding of biology ${ }^{20}$ eröffne. Damit steht der Grundgedanke im Raum, dass der Weg zum Verständnis des Komplexen über die Reduktion auf die Bestandteile der niedrigeren Ebene verlaufe:Wenn für jedes Gen die molekulare und biologische Funktion bekannt ist, wie es Craig Venter in seiner Vision der Minimalzelle formuliert, dann eröffne sich damit neues Wissen über das übergeordnete Phänomen, die

\footnotetext{
${ }^{19}$ Hutchison III et al., Design and synthesis, aad 6253-1.

${ }^{20}$ Schwille, Bottom-up Synthetic Biology, 1252, mit Bezug auf B.J. Yeh/W.A. Lim, Synthetic biology: lessons from the history of synthetic organic chemistry, Nature Chemical Biology 3 (2007), 521-525.
} 
Zelle, die oft als kleinste lebendige Einheit angesehen wird und damit repräsentativ für das Phänomen des Lebendigen in der Biologie steht.

Die Orientierung am "rational design" ist die methodische Entsprechung dazu: Statt einzelne künstliche Komponenten in ein natürliches System zu übertragen, wie es etwa bei transgenen Organismen der Fall ist, ist das Ziel eine vom Reissbrett und unter menschlicher Kontrolle synthetisierte Zelle, die sowohl in der Synthese als auch in der späteren Performanz ihrer (Lebens-) Funktionen keine Zufallselemente mehr enthält - eben eine "Biomaschine". Dies ist die radikale Vision der Synthetischen Biologie, die reduktionistische Letzterklärungsansprüche heraufbeschwört, zumindest wenn das Maschinenhafte der Biomaschinen als Kern der belebten Natur ausgemacht wird.

\section{3. »Synthetische Biologie - die Synthese der Biologie ${ }^{21}$ ?}

Die Argumentation, die Konstruktion »lebender Biomaschinen" führe zu einem lückenlosen Verständnis der Grundlagen der Biologie, wirft offensichtlich mehrere Fragen auf. $\mathrm{Zu}$ nennen wäre die Unhaltbarkeit dieses Anspruchs angesichts derVielfalt der biologischen Fragestellungen, die es unmöglich machen, von den Grundlagen »der Biologie« zu sprechen - die Biologie stellt vielfältige Fragen, die nicht alle eine molekularbiologische Antwort nach sich ziehen. Ebenso zeigt sich, dass mit der Vielfalt der Forschungsfragen eine Differenzierung des Konzepts des Lebendigen einhergeht: mal überwiegt die Frage nach dem phylogenetischen Zusammenhang aller Lebewesen, ${ }^{22}$ ein anderes Mal interessiert die Konstitution des einzelnen Lebewesens in funktionaler Hinsicht, oder es werden scheintote Überdauerungsformen ins Weltall geschickt, um etwas über die Über-Lebenskraft einzelner biologischer Arten herauszufinden. Es gibt kein Einverständnis, wie die Grenzen des biologischen Forschungsobjekts zu bestimmen sind, und so kann ein solches Einverständnis auch nicht durch die Konstruktion einer Minimalzelle - quasi als Repräsentantin der lebendigen Natur - geschaffen werden.

${ }^{21}$ Titel des bereits genannten Papers von Ausländer et al., Synthetische Biologie.

${ }^{22}$ Elisabeth Loos, Biologische Konzepte von `Leben und die Synthetische Biologie, in: Leben konstruieren? Deutungsmuster Synthetischer Biologie, hg. v. Tobias Grassmann/Stefanie Herresthal, TTN edition 1/2015, 29-47. 
Auch eine grundlegende Kritik reduktionistischer Deutungsansprüche etwa auf die Bestimmung »des Lebendigen" oder »der Biologie" hat ihre Berechtigung, doch möchte ich an dieser Stelle darauf hinweisen, dass sie sich oft auf den zugespitzten weltanschaulichen Teil des Forschungsfeldes konzentriert, das nicht unbedingt als repräsentativ angesehen werden kann. Das Entlarven von reduktionistischen Zuspitzungen - die es zweifelsfrei gibt - als letztlich metaphysische Aussagen, die sich ihrer Voraussetzungshaftigkeit nicht bewusst sind, sollte meines Erachtens nicht den Umgang mit der Naturwissenschaft dominieren, ebenso wenig wie der Verweis auf Lücken der naturwissenschaftlichen Forschung, seien sie prinzipieller Art wie beim Ich-Bewusstsein des Subjekts ${ }^{23}$ oder vermutlich vorläufiger Art wie die 150 ungeklärten Gene der Minimalzelle Craig Venters, die zu Beginn erwähnt wurden.

Denn bei genauerem Hinsehen zeigt sich meines Erachtens, dass sich die Synthetische Biologie gar nicht so gut für die Konstruktion eines naturwissenschaftlich-reduktionistischen Weltbildes eignet, wie man aus den zuvor zitierten Äusserungen ableiten könnte und wie es mitunter in der Sekundärliteratur und der medialen Berichterstattung, aber auch von Akteuren der Synthetischen Biologie selbst dargestellt wird - als wäre mit dem Gelingen der synthetisch-biologischen Forschungsprojekte unmittelbar der Anspruch verbunden, die weltanschauliche Deutungshoheit über das Leben zu erlangen und den Naturalismus vom Status der Metaphysik in ein empirisch belegtes Faktum zu verwandeln.

Unterstreichen möchte ich diese These mit verschiedenen Beobachtungen.

Zunächst gilt es festzuhalten, dass die konkreten Methoden der synthetisch-biologischen Forschung nicht auf das vollständig rationalisierte "design from scratch" reduziert werden können. Damit möchte ich nicht auf eine praktische Uneinholbarkeit der selbstgesetzten Ideale der Synthetischen Biologie abheben, sondern darauf hinweisen, dass es Methoden gibt, die gar nicht das Ziel einer vollständigen Rationalisierung beinhalten, etwa im Bereich der »directed evolution «. ${ }^{24}$ Bei dieser weit verbreiteten Forschungsmethode wird zunächst im Labor eine Vielzahl von Mutanten einer Gensequenz erzeugt; das ist die sogenannte Mutagenese, die hervorgerufen wird z.B. durch das Einbringen von Transposons. Diese Mutagenese ist nicht kontrollierbar, sondern setzt auf das Entstehen einer grossen

\footnotetext{
${ }^{23}$ Damit verknüpft ist auch die Qualia-Debatte, die nach wie vor mit grossem Engagement gefuihrt wird.

${ }^{24}$ Siehe dazu etwa Ausländer et al., Synthetische Biologie, 6500-6502.
} 
Anzahl zufälliger Mutationen, die dann auf ihre Funktionen in der Zelle unter bestimmten Bedingungen getestet werden (screening), etwa indem sie mit bestimmten Antibiotika konfrontiert werden und getestet wird, ob eine neue Resistenz entstanden ist. Mehrere Zyklen von Mutagenese und Screening, zum Teil weit über 100 an der Zahl, werden hintereinander durchgeführt, sodass eine Evolution im Schnelldurchlauf entsteht, die mit der natürlichen Evolution teilt, dass sie ebenfalls von "Chance and Necessity ${ }^{25}$ bestimmt wird. Die Methode liefert gute Ergebnisse und ist in der Biotechnologie weit verbreitet - und unterscheidet sich durch die gezielte Aufnahme von nicht-steuerbaren Zufallselementen im Grundgedanken deutlich vom ebenfalls praktizierten "rational design«. Eine gute Konstruktion braucht demnach nicht notwendigerweise lückenloses Wissen über ihre Grundlagen und führt auch nicht automatisch dahin.

Das spiegelt sich auch darin, dass Forscherinnen und Forscher der Synthetischen Biologie ihre Arbeit selbst als "tinkering in the lab" bezeichnen - ohne dies despektierlich zu meinen - und somit die oben zitierte Aussage relativieren, das "tinkering" der Natur solle durch das »engineering" im Labor ersetzt werden. Ein Vorgehen nach dem »trial and error «-Prinzip ist durchaus normal und reflektiert das Bewusstsein von der Komplexität biologischer Zusammenhänge. ${ }^{26}$ Das Besondere daran ist meines Erachtens, dass dies nicht als Eingeständnis eines Defizits an Wissenschaftlichkeit zu verstehen ist, sondern Ausdruck einer pragmatisch orientierten Suche der besten Lösung für eine bestimmte Aufgabe. Wenn sich über das »trial and error«-Prinzip eine funktionierende Lösung anbietet, besteht nicht unbedingt ein grundsätzliches Interesse an der Aufklärung der kausalen Wirkmechanismen. Das Motto "What I cannot create, I cannot understand " ist somit zumindest in seiner Geltung für die gesamte Synthetische Biologie nicht haltbar.

In diesem Sinne ist auch auf eine Differenzierung des oben dargestellten »engineering«-Begriffs zu drängen, der nicht ohne Weiteres auf vollständige wissenschaftliche Rationalisierbarkeit enggeführt werden kann, wenn man dem Technikphilosophen Günther Ropohl folgt, der in seiner Darstellung der "Allgemeinen Technologie» Kog-

\footnotetext{
${ }^{25}$ So bestimmte der Biologe Jacques Monod im gleichnamigen Buch die beiden Triebkräfte der Evolution. Jacques Monod, Chance and Necessity: An Essay on the Natural Philosophy of Modern Biology, New York 1971.

${ }^{26}$ Auf den kreativen Anteil (»bricolage «) der synthetisch-biologischen Forschungen geht u.a. ein: Leona Litterst, Synthetic Biology - Playing Games?, in: Ambivalences of Creating Life. Societal and Philosophical Dimensions of Synthetic Biology, hg. v. Margret Engelhard/Kristin Hagen/Georg Toepfer, Cham 2016, 243-250.
} 
nition und Invention in keinen zwingenden Zusammenhang stellt. ${ }^{27}$ Auch Ingenieure basteln, »engineering « und »tinkering « liegen nicht so weit auseinander, könnte man etwas verkürzt festhalten, und umgekehrt gibt es keinen zwingenden Zusammenhang zwischen einer gelungenen Synthese und einem besseren Verständnis ihrer Grundlagen: »What I cannot understand, I nevertheless can try to construct«, wäre die passendere Losung.

Darüber hinaus wurde schon darauf hingewiesen, dass es gerade der »engineering «-Anteil der Synthetischen Biologie ist, der sie als einen pragmatischen, d.h. handlungsorientierten statt erkenntnisorientierten Umgang mit biologischen Komponenten ausweist, indem diese als "black box" gesehen und nichtsdestotrotz praktisch verwendet werden. ${ }^{28}$ Dies zeigt deutlich, dass die Synthetische Biologie nicht als theoretische Grundlagenwissenschaft mit der Absicht einer abschliessenden Theorie der belebten Natur zu verstehen ist. Die bereits genannte Minimalzellenforscherin Petra Schwille spricht selbst an, dass ihre Forschungen nur ein begrenztes Potenzial »for truly understanding living systems " hätten. ${ }^{29}$

Die Integration nicht-rationalisierter Elemente in die Synthetische Biologie sollte daher nicht als konzeptionelles Defizit verstanden werden, sondern als Ausdruck eines heuristisch orientierten Forschungspragmatismus, der wissenschaftstheoretisch schon seit längerem als ein Kennzeichen von Naturwissenschaft reflektiert wird. Anführen möchte ich hier William Wimsatt, der die Erarbeitung von "key heuristics" als Aufgabe einer realistischen "philosophy for science" sieht und stets die Fehleranfälligkeit des Menschen im Blick hat, den er als "limited being " charakterisiert; seine naturalistische Methodologie kann etwa Reduktion als eine methodische Notlösung der Wissenschaft zulassen, ohne jedoch für einen Reduktionismus zu plädieren. Wimsatt geht soweit, der heuristischen Vielfalt eine ontologische Vielfalt zuzuordnen: er nennt dies "rainforest ontology", die er im Gegensatz zu einer minimalistischen "desert ontology" (in Anspielung auf Willard Quine) sieht, die sich um die Rückführung aller Phänomene auf eine kleinstmögliche Anzahl

\footnotetext{
${ }^{27}$ Günter Ropohl, Allgemeine Technologie. Eine Systemtheorie der Technik, Karlsruhe ${ }^{3} 2009,23.258 f$.

${ }^{28}$ Siehe dazu etwa Michael Bölker, Revolution der Biologie? Ein Überblick über die Voraussetzungen, Ansätze und Ziele der Synthetischen Biologie, in: Was ist Leben - im Zeitalter seiner technischen Machbarkeit? Beiträge zur Ethik der Synthetischen Biologie, hg. v. Peter Dabrock/Michael Bölker/Matthias Braun/Jens Ried, Freiburg/ München 2011, 35-37.

${ }^{29}$ Schwille, Bottom-up Synthetic Biology, 1253.
} 
ontologischer Bestimmungen konzentriere. ${ }^{30}$ Ian Hacking setzt in seiner "Einführung in die Philosophie der Naturwissenschaften" andere Akzente, doch auch er bekennt freimütig, dass er die meisten Rationalitätsfragen verwerfe und stattdessen einen Realismus vertrete, der auf den "allerpragmatischsten Gründen $\aleph^{31}$ beruhe und ohne eine Korrespondenztheorie derWahrheit auskomme ${ }^{32}$. Realität steht laut Hacking "in engerem Zusammenhang mit dem, was wir in der Welt tun, als mit dem, was wir über sie denken. ${ }^{33}$ Als letztes Beispiel sei Stephan Fischer genannt, der mit Bezug auf die Synthetische Biologie feststellt, dass sich in ihren Forschungen zeige, wie in naturwissenschaftlichen Modellen eine "modale Umkehrung" stattfinde: Tatsachen stünden nicht länger im Status des Actualen, das potenziell Anwendungen nach sich ziehen kann, sondern umgekehrt sei es so, dass mithilfe von Modellen die Anwendungen einen actualen Status einnehmen, aus denen verschiedene, potenzielle Tatsachen folgen können. ${ }^{34}$

Habe ich also einen reduktionistischen Pappkameraden der Biotechnologie aufgestellt, um ihn dekonstruieren zu können? Das trifft mein Anliegen nicht vollständig, auch wenn ich dem Pappkameraden durchaus ein wenig zusetzen möchte, weil er eben doch immer wieder auftaucht. Im Vordergrund steht jedoch die Absicht aufzuzeigen, dass eine Entscheidung für oder gegen eine reduktionistische Weltanschauung für den Erfolg der modernen Biotechnologie nicht ausschlaggebend ist - die Weltbehandlung ist der Weltanschauung vorgeordnet, und so bildet sich ein dynamisches und flexibles Gerüst der wechselseitigen Verbindungen im naturwissenschaftlichen Forschen. Bezüge auf "die Natur« oder »das Leben« können und sollen kritisch reflektiert werden, ohne den korrespondierenden Konzepten zu viel Bedeutung zu schenken. Denn gerade die modernen Biotechnologien, denen so oft die endgültige Entzauberung der Welt zugeschrieben wird, eignen sich dafür aufgrund ihres tiefverankerten Pragmatismus, der über die Heuristik selten hinausgeht, nur bedingt.

Auch der Dialog zwischen "religion and science« sollte sich daher meines Erachtens nicht nur auf die Kritik des weltanschaulich-

\footnotetext{
${ }^{30}$ William C. Wimsatt, Re-Engineering Philosophy for Limited Beings. Piecewise Approximations to Reality, Cambridge, MA/London 2007.

${ }^{31}$ Ian Hacking, Einfuihrung in die Philosophie der Naturwissenschaften, Stuttgart 1996, 15.

32 Ebd., 39.

${ }^{33}$ Ebd., 40.

${ }^{34}$ Stephan M. Fischer, Modale Umkehrung. Wissenschaftstheorie, Werte und die Synthetische Biologie, in: Was ist Leben, hg. v. Peter Dabrock et al., 227-250.
} 
zugespitzten Naturalismus konzentrieren, sondern zunächst einmal wahrnehmen, dass die Naturwissenschaft, zumal die angewandte, ein instrumentalistisches Verhältnis zu ihren Methoden hat - was funktioniert, ist akzeptiert - ohne aus der Heuristik gleich in ontologische oder metaphysische Letztaussagen überzugehen. Was dabei entsteht, sind vorläufige Modelle über Ausschnitte der Wirklichkeit, die keinen philosophischen Realismus begründen, sondern maximal eine realistische "philosophy for science». Dies ist jedoch nicht mit philosophischer Zahnlosigkeit gleichzusetzen, denn es ist offensichtlich, dass auch auf der Basis von pragmatischer Weltbehandlung Aussagen und Modelle über den Menschen und die Welt entstehen - vorläufige, sich wandelnde zwar, aber dennoch sehr wirkkräftige.

- Elisabeth Loos ist Wissenschaftliche Mitarbeiterin am Institut für Systematische Theologie der Martin-Luther-Universität Halle-Wittenberg. In ihrer Dissertation wird das Verhältnis von biologischen und theologischen Lebenskonzepten im Kontext von Forschungen der Synthetischen Biologie untersucht. Weitere Forschungsinteressen sind der Dialog von Natur- und Geisteswissenschaften sowie der Neue Atheismus. 